\title{
Electron Localization in Defective Ceria Films: A Study with Scanning-Tunneling Microscopy and Density-Functional Theory
}

\author{
Jan-Frederik Jerratsch, ${ }^{1}$ Xiang Shao, ${ }^{1}$ Niklas Nilius, ${ }^{1, *}$ Hans-Joachim Freund, ${ }^{1}$ Cristina Popa,${ }^{2}$ \\ M. Veronica Ganduglia-Pirovano, ${ }^{2, \dagger}$ Asbjörn M. Burow, ${ }^{2}$ and Joachim Sauer ${ }^{2, \dagger}$ \\ ${ }^{1}$ Fritz-Haber-Institut der MPG, Faradayweg 4-6, 14195 Berlin, Germany \\ ${ }^{2}$ Humboldt-Universität zu Berlin, Unter den Linden 6, 10099 Berlin, Germany
}

(Received 5 January 2011; published 14 June 2011)

\begin{abstract}
Scanning-tunneling microscopy and density-functional theory have been employed to identify the spatial correlation between an oxygen vacancy and the associated $\mathrm{Ce}^{3+}$ ion pair in a defective $\mathrm{CeO}_{2}(111)$ film. The two $\mathrm{Ce}^{3+}$ ions can occupy different cationic shells around the vacancy. The resulting variation in the chemical environment leads to a splitting of the filled $\mathrm{Ce}^{3+} f$ levels, which is detected with STM spectroscopy. The position of the $\mathrm{Ce}^{3+}$ ion pair is reflected in characteristic defect patterns observed in empty-state STM images, which arise from the bright appearance of $\mathrm{Ce}^{4+}$ ions next to the defect while the $\mathrm{Ce}^{3+}$ remain dark. Both findings demonstrate that at least one excess electron localizes in a Ce ion that is not adjacent to the $\mathrm{O}$ vacancy.
\end{abstract}

DOI: 10.1103/PhysRevLett.106.246801

Ceria is a widely used oxide support for several oxidation reactions, such as the water-gas shift and $\mathrm{NO}_{x}$ reduction [1-3]. The outstanding performance of ceria in promoting oxidation processes relies to a large extent on its facile reducibility and the associated ability to release lattice oxygen [4]. The vacancies are easily healed again in an O-rich ambience, which makes the oxide perfectly suited to balance the oxygen supply during a catalytic reaction. On removing an $\mathrm{O}$ atom, two electrons are left behind in the lattice that form two reduced $\mathrm{Ce}^{3+}$ species [4]. The electrons occupy split-off states of the initially empty Ce $4 f$ band, lying inside the $\mathrm{O}_{2 p}-\mathrm{Ce}_{5 d}$ band gap of ceria and being highly localized in space.

The reduction of ceria can be followed with photoelectron spectroscopy, by using the presence of filled Ce $4 f$ states or characteristic shifts in the Ce $3 d$ core levels as a measure $[5,6]$. This approach provides only spatially averaged information, and the localization of the $\mathrm{O}$ vacancies and associated $\mathrm{Ce}^{3+}$ ion pairs is not revealed. In contrast, scanning-tunneling (STM) and atomic force microscopy have been successfully employed to identify single $O$ vacancies in surface or subsurface ceria layers $[7,8]$. However, no information on the electronic properties of the defects was acquired in this case, and the question of electron localization remained unsolved. Early densityfunctional theory (DFT) calculations assumed a stabilization of the extra electrons in Ce ions next to the defect $[7,9,10]$, while recent studies suggested the occupancy of more distant cationic sites [11-13]. In ceria nanoclusters, the $\mathrm{Ce}^{3+}$ ions were found to form preferentially at lowcoordinated edge sites, even if the $\mathrm{O}$ vacancy is far away [14]. Those differences demonstrate the sensitivity of electron-localization phenomena on the computational approach.
PACS numbers: 73.20.Hb, 68.37.Ef, 71.15.Mb, 73.61.Ng

In this work, we use a combined STM and DFT approach to provide insight into the spatial correlation between $\mathrm{O}$ defects and the associated $\mathrm{Ce}^{3+}$ ion pair in a $\mathrm{CeO}_{2}(111)$ surface. We demonstrate that electrons left behind upon vacancy formation occupy $\mathrm{Ce}$ sites that are not in a nextneighbor position to the defect. This possibility to explore electron-localization effects opens a direct route to monitor oxidation and reduction processes in the catalytically relevant ceria system [15].

The experiments have been performed in an ultrahigh vacuum STM operated at $10 \mathrm{~K}$. The oxide film is prepared by depositing $\mathrm{Ce}$ onto a sputtered and annealed $\mathrm{Ru}(0001)$ surface in $5 \times 10^{-6} \mathrm{mbar}_{2}$, whereby half of the material is dosed at $100 \mathrm{~K}$ and half at $450 \mathrm{~K} \mathrm{[16].} \mathrm{After} \mathrm{annealing} \mathrm{to}$ $1000 \mathrm{~K}$ in $\mathrm{O}_{2}$, the film displays a sharp $(\sqrt{ } 2 \times \sqrt{ } 2)$ LEED pattern that is characteristic for the $\mathrm{CeO}_{2}$ (111) surface. The mean film thickness is adjusted to five O-Ce-O trilayers. Highly resolved STM measurements are mainly obtained at positive sample bias (unoccupied states), indicating that the $\mathrm{Ce}^{4+}$ ions are responsible for the contrast (Fig. 1). At negative bias, the filled O $2 p$ orbitals govern the contrast; however, atomic resolution is more difficult to obtain. In both cases, the observed atom periodicity of $4.0 \AA$ is in line with the $\mathrm{CeO}_{2}$ lattice constant of $3.88 \AA$.

Ceria films are prepared in an excess of $\mathrm{O}_{2}$ and exhibit only a small defect concentration. Oxygen vacancies are thus generated by injecting $50 \mathrm{eV}$ electrons ( $1 \mathrm{mC}$ dose) at $100 \mathrm{~K}$. Because of the highly nonthermal formation process, the defects are not expected to be in an equilibrium distribution; however, most of them are located in the oxide surface given the low excitation energies. At positive bias, they appear as paired and tripled protrusions of $\sim 0.4 \AA$ height, which mark the $\mathrm{Ce}^{4+}$ ions around the vacancy as shown below [Figs. 1(d) and 1(e)]. At negative polarity, 


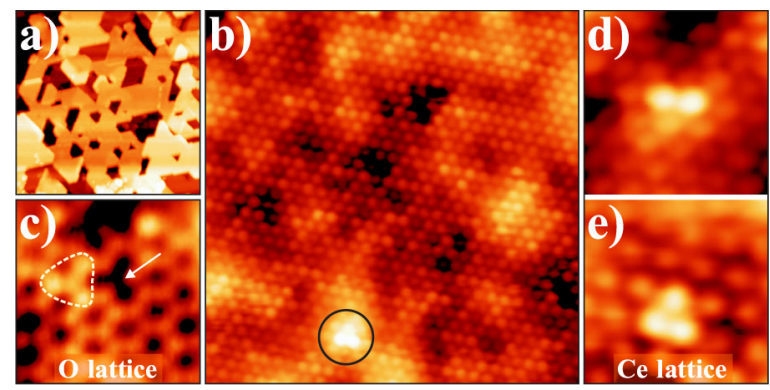

FIG. 1 (color online). (a) STM image of a five trilayer thick $\mathrm{CeO}_{2}$ film grown on $\mathrm{Ru}(0001)\left(3.1 \mathrm{~V}, 100 \times 100 \mathrm{~nm}^{2}\right)$. (b) Atomically resolved image showing the $\mathrm{Ce}$ sublattice $\left(1.2 \mathrm{~V}, 12 \times 12 \mathrm{~nm}^{2}\right)$. The protrusion in the lower part is assigned to a single $\mathrm{O}$ vacancy. (d),(e) Similar defects imaged with a tip configuration that is sensitive to the Ce lattice $(1.2 \mathrm{~V}$, $\left.2.4 \times 2.4 \mathrm{~nm}^{2}\right)$ and (c) to the $\mathrm{O}$ lattice $(-2.2 \mathrm{~V})$. The arrow in (c) marks a surface $\mathrm{O}$ vacancy, while the triangle denotes a subsurface defect characterized by three protruding $\mathrm{O}$ ions.

they transform into atom-sized holes, confirming their identity as a surface $\mathrm{O}$ vacancy [Fig. 1(c), arrow] [7,8]. Also, subsurface vacancies that appear as three protruding maxima at negative bias are occasionally observed but have been disregarded in this study [Fig. 1(c), dashed triangle].

The electronic properties of surface defects are analyzed with STM conductance spectroscopy performed with the lock-in technique $(10 \mathrm{mV} / 1073 \mathrm{~Hz})$. The stoichiometric film exhibits a band gap ranging from -2.5 (valence band) to $+3.5 \mathrm{~V}$ (conduction band) [Fig. 2(a)]. The gap size of
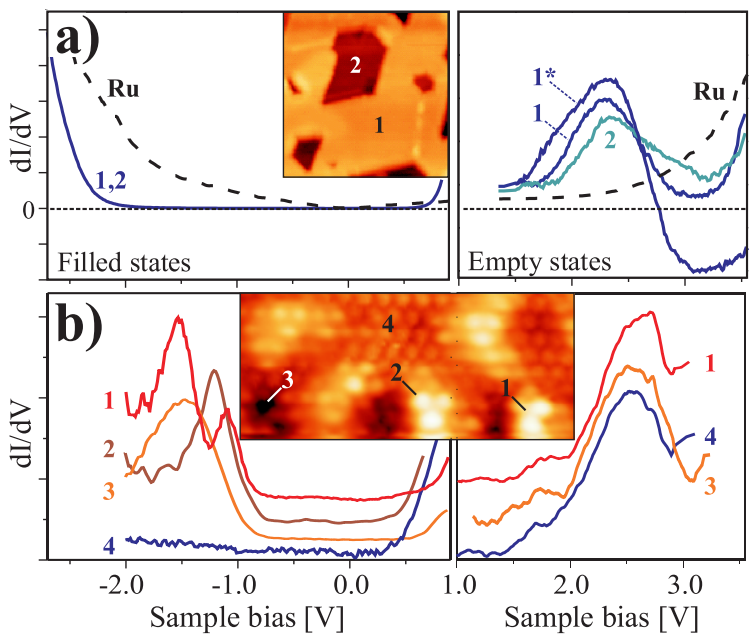

FIG. 2 (color online). Conductance spectra of (a) stoichiometric and (b) reduced $\mathrm{CeO}_{2}(111)$ taken at negative (left) and positive bias (right). The spectral positions are marked in the insets (a) $2.0 \mathrm{~V}, 15 \times 15 \mathrm{~nm}^{2}$; (b) $1.2 \mathrm{~V}, 8 \times 4 \mathrm{~nm}^{2}$. All curves have been taken with $5 \mathrm{pA}$ current, apart from $1 *(50 \mathrm{pA})$. While the $d I / d V$ maximum at $2.3 \mathrm{~V}$ reflects the empty Ce $4 f$ band, the peaks around $-1.5 \mathrm{~V}$ are assigned to filled $f$ states.
$6.0 \mathrm{eV}$ is close to the bulk value obtained from electronenergy loss and optical spectroscopy [5]. An extra $d I / d V$ peak at $2.3 \mathrm{eV}$ is detected inside the gap and assigned to the unoccupied $\mathrm{Ce} 4 f$ band for the following reasons. Its energy position fits well to the $f$ band center derived from electron-energy loss and inverse photoelectron spectroscopy $[5,17]$. The peak is visible only at small tipsample distances, adjusted with a bias set point close to the conduction band onset, as expected for a highly contracted electronic state. And a negative-differential resistance behavior is revealed for higher set-point currents $(I>50 \mathrm{pA})$, as reflected in the negative $d I / d V$ signal beyond the peak [Fig. 2(a), spec 1*]. The negativedifferential resistance effect originates from the loss of conductivity when the $f$-related transport channel closes in the spectral run. It is symptomatic for electron transport through spatially and energetically confined states $[18,19]$ and therefore in line with our assignment of the gap states to the empty $4 f$ band.

On defective ceria, also filled $f$ states should appear at the position of the $\mathrm{Ce}^{3+}$ ions $[4,20]$. Their detection is challenging due to the small tip overlap with the localized states in the gap. Sufficient spectral sensitivity is achieved only if the tip is stabilized inside the empty $f$ band and then ramped to negative bias [Fig. 2(b), left]. Whereas no filled states are observed on defect-free oxide patches (spec 4), new $d I / d V$ peaks appear at around $-1.5 \mathrm{~V}$ in the vicinity of surface vacancies. The signal intensity strongly depends on a precise positioning of the tip above the defect and quickly drops to zero if the tip is displaced by more than $5 \AA$. Also, the spectral shape changes when going from one defect to another (specs 1-3). Whereas defects appearing as a triple protrusion always display a single peak at around $-1.2 \mathrm{~V}$ (spec 2), a splitting with maxima at -1.5 and $-1.1 \mathrm{~V}$ is revealed for certain paired protrusions (spec 1). However, the latter also appear with only one peak in the conductance spectra. We note that similar gap states are detected for other defect types, such as voids in the $\mathrm{Ce}$ lattice (spec 3 ) and step edges. Common to all defects is an empty-state spectrum that is similar to the one of the stoichiometric film [Fig. 2(b), right].

The high spatial confinement and energy position of the gap states suggest a relation to the filled $4 f$ levels localized at the $\mathrm{Ce}^{3+}$ ions, as similar states were detected with electron-energy loss and photoelectron spectroscopy before $[5,15]$. However, a conclusive interpretation requires DFT calculations and have been performed at the PBE + $U$ level $(U=4.5 \mathrm{eV})$, by using the VASP code with a $400 \mathrm{eV}$ cutoff for the plane-wave basis set [21]. Atomic cores are described by the projected augmented wave method. The $\mathrm{O}$ vacancy is positioned in the top layer of the $(3 \times 3)$ supercell representing the $\mathrm{CeO}_{2}(111)$, while all cationic sites of the slab are considered as potential positions for the $\mathrm{Ce}^{3+}$ ions. The electronic structure is deduced from a $(2 \times 2 \times 1) k$-point sampling of the Brillouin zone. 
TABLE I. Geometric structure, $\mathrm{Ce}^{3+}$ coordination numbers, vacancy formation energy $\left(E_{\mathrm{def}}\right)$ with respect to $\frac{1}{2} \mathrm{O}_{2}$, and splitting of occupied $f$ states for different $\mathrm{O}$ vacancy $/ \mathrm{Ce}^{3+}$ configurations. The ion with the larger $\mathrm{CN}$ has the lower $f$ state energy.

\begin{tabular}{|c|c|c|c|c|c|}
\hline $\begin{array}{l}\mathrm{Ce}^{3+} \\
\text { configuration } \\
\end{array}$ & $\begin{array}{c}\mathrm{Ce}^{3+}-\mathrm{Ce}^{3+} \\
\text { distance }[\AA]\end{array}$ & $\begin{array}{l}\mathrm{Ce}^{3+} \text {-defect } \\
\text { distances }[\AA]\end{array}$ & $\begin{array}{c}\text { Coordination } \\
\text { numbers of } \mathrm{Ce}^{3+}\end{array}$ & $E_{\text {def }}\left(\frac{1}{2} \mathrm{O}_{2}\right)[\mathrm{eV}]$ & $\begin{array}{c}f \text { state } \\
\text { splitting }[\mathrm{eV}]\end{array}$ \\
\hline$\overline{2_{1}-2_{1}}$ & 3.91 & $4.52 ; 4.52$ & $7 ; 7$ & $2.06^{\mathrm{a}}$ & 0.00 \\
\hline $1_{1}-2_{1}$ & 3.90 & $2.54 ; 4.53$ & $6 ; 7$ & $2.16^{\mathrm{a}}[11]$ & 0.05 \\
\hline $1_{1}-1_{1}$ & 4.17 & $2.54 ; 2.54$ & $6 ; 6$ & $2.22^{\mathrm{a}}[11]$ & 0.00 \\
\hline $1_{1}-3_{1}$ & 3.74 & $2.51 ; 5.95$ & $6 ; 7$ & $2.28^{\mathrm{a}}$ & 0.10 \\
\hline $1_{1}-4_{2}$ & 5.43 & $2.51 ; 7.08$ & $6 ; 8$ & 2.45 & 0.47 \\
\hline $2_{2}-4_{2}$ & 3.90 & $4.47 ; 7.08$ & $8 ; 8$ & 2.59 & 0.06 \\
\hline
\end{tabular}

${ }^{\text {a }}$ Similar defect-formation energies have been calculated for a $3 \times 4$ cell in Ref. [12].

We find six configurations of the $\mathrm{Ce}^{3+}$ ion pair with defectformation energies below $2.6 \mathrm{eV}$ (Table I). The $\mathrm{Ce}^{3+}$ positions are hereby labeled in an $n_{m}$ scheme, with $m$ being the cationic layer counted from the surface and $n$ denoting the shell of cations surrounding the vacancy. For example, $\mathbf{2}_{\mathbf{1}}$ describes a position in the surface Ce layer that is second neighbor to a defect. The properties of the $\mathrm{Ce}^{3+}$ ions are now governed by the number of $\mathrm{O}$ ligands, i.e., the coordination number $(\mathrm{CN}) \cdot \mathrm{A} \mathrm{Ce}^{3+}$ in the surface trilayer has seven and six $\mathrm{O}^{2-}$ neighbors if it sits in a second- $\left(\mathbf{2}_{\mathbf{1}}\right.$ site $)$ and first-neighbor position $\left(\mathbf{1}_{\mathbf{1}}\right.$ site) to the defect, respectively, but eight in a bulk environment. There are two configurations in Table I with equal $\mathrm{CN}$ for the two $\mathrm{Ce}^{3+}$ ions, namely, the $\mathbf{2}_{\mathbf{1}}-\mathbf{2}_{\mathbf{1}}$ and $\mathbf{1}_{\mathbf{1}}-\mathbf{1}_{\mathbf{1}}$ arrangements. In both cases, the two ions have filled $f$ orbitals with the same energy as they experience the same chemical environment. For $\mathrm{Ce}^{3+}$ ions with different CNs, we find a splitting of the $f$ orbitals due to the different distances from the vacancy. The splitting is of the order of $0.1 \mathrm{eV}$ if the $\mathrm{CN}$ differs by one (as in $\mathbf{1}_{\mathbf{1}}-\mathbf{2}_{\mathbf{1}}$ and $\mathbf{1}_{\mathbf{1}}-\mathbf{3}_{\mathbf{1}}$ pairs) but increases to $0.5 \mathrm{~V}$ for a $\mathrm{CN}$ mismatch of two (Fig. 3). The latter case occurs in the $\mathbf{1}_{\mathbf{1}}-\mathbf{4}_{\mathbf{2}}$ pair that consists of a sixfold and an eightfold coordinated $\mathrm{Ce}^{3+}$ ion in the surface and subsurface layer,
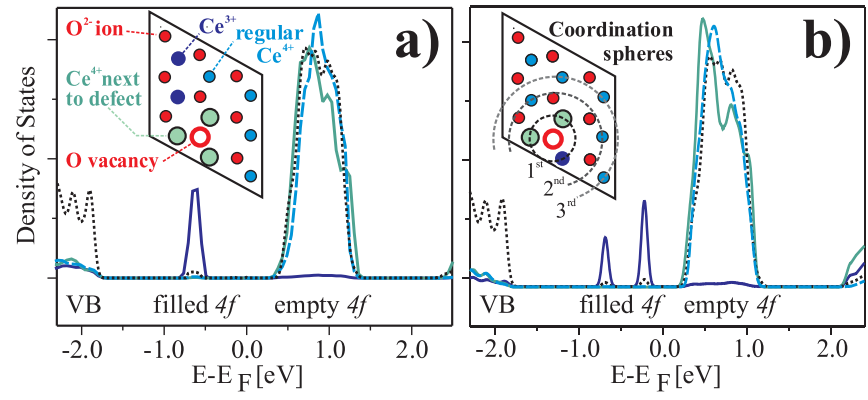

FIG. 3 (color online). Total and atom-projected density of states for (a) the energetically favored $\mathbf{2}_{\mathbf{1}}-\mathbf{2}_{\mathbf{1}}$ and (b) the $\mathbf{1}_{\mathbf{1}}-$ $\mathbf{4}_{2} \mathrm{Ce}^{3+}$ ion pair. Dark solid line: $\mathrm{Ce}^{3+}$; bright solid line: $\mathrm{Ce}^{4+}$ next to an $\mathrm{O}$ vacancy; dashed line: other surface $\mathrm{Ce}^{4+}$; and dotted line: total density of states. All spectra are normalized with respect to the number of Ce ions. The respective unit cells and defect coordination spheres are shown in the insets. respectively. From those results, it becomes clear why the experimental spectra show either a double- or a single-peak structure. The two $\mathrm{Ce}^{3+}$ ions in spec 1 of Fig. 2(b) are apparently located in rather different environments, while the pair in spec 2 has a comparable $\mathrm{CN}$. The localization of the $\mathrm{Ce}^{3+}$ ions with respect to the defect is not deduced from the splitting that is sensitive only to differences in the local environment.

This information is, however, contained in empty-state STM topographies, which also reflect the local electronic structure of the ceria surface. We have therefore simulated
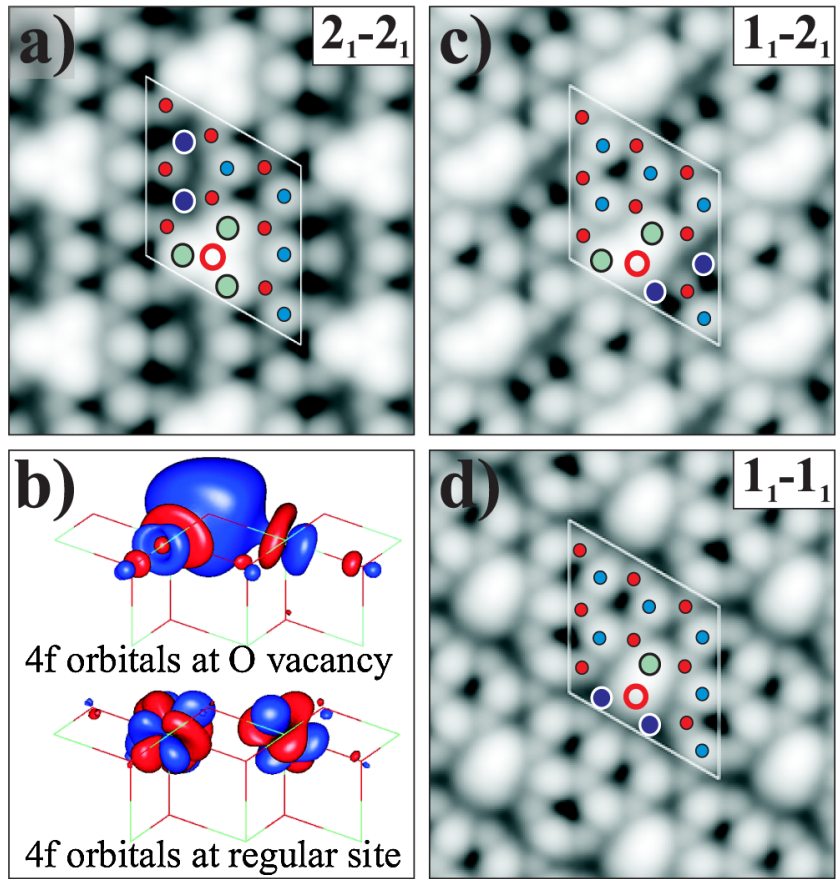

FIG. 4 (color online). DFT simulations of empty-state STM images ( $0-2 \mathrm{eV})$. Small blue dots denote the $\mathrm{Ce}^{4+}$ ions, red dots the $\mathrm{O}^{2-}$, and the red circle the $\mathrm{O}$ vacancy. While $\mathrm{Ce}^{3+}$ ions (large blue circles) are nearly invisible, $\mathrm{Ce}^{4+}$ ions next to the $\mathrm{O}$ defect appear brighter. This results in different patterns depending on the number of $\mathrm{Ce}^{4+}$ ions around the defect. The origin of the contrast is the larger expansion of the $\mathrm{Ce}^{4+} 4 f$ orbitals in presence of the vacancy (b). 
STM images for bias voltages, where electron tunneling into the empty Ce $4 f$ band is enabled (Fig. 4). The $\mathrm{Ce}^{3+}$ (large blue dots) are not visible in this case, because their empty $4 f$ states shift to higher energies due to the better screening of the Ce core potential in the electron-rich ions (Fig. 3). The $\mathrm{Ce}^{4+}$ ions, on the other hand, appear bright especially if they are adjacent to an $\mathrm{O}$ defect (large green dots). The reason for the enhanced contrast of the latter has been clarified with cluster calculations for the $\mathbf{2}_{\mathbf{1}} \mathbf{-} \mathbf{2}_{\mathbf{1}}$ ion pair, performed with PBE0 hybrid functionals and an electrostatic embedding method [22]. Because of the missing repulsive potential at the $\mathrm{O}$ vacancy site, the $4 f$ orbitals largely extend towards the empty space and partially delocalize over the $\mathrm{Ce}^{4+}$ ions surrounding the defect [Fig. 4(b)]. Tunneling into those expanded states gives rise to the bright appearance of the $\mathrm{Ce}^{4+}$ ions next to the vacancy. This interpretation allows us to draw conclusions on the position of the excess electrons with respect to the $\mathrm{O}$ defect. If the vacancy is surrounded only by $\mathrm{Ce}^{4+}$ ions and the $\mathrm{Ce}^{3+}$ occupy more distant coordination shells, a symmetric triple protrusion becomes visible in empty-state images [Fig. 4(a)]. This triple signature is indeed observed in the STM, although its abundance is relatively small [Fig. 1(e)]. The most frequently seen defect type is the paired protrusion, which is readily assigned to two $\mathrm{Ce}^{4+}$ localized next to an $\mathrm{O}$ vacancy [Figs. 1(d) and 4(c)]. The third faint species marks a $\mathrm{Ce}^{3+}$ in a next-neighbor position to the defect, while the second $\mathrm{Ce}^{3+}$ cannot be identified in the image. The last possible configuration reveals a single maximum and reflects an $\mathrm{O}$ vacancy surrounded by one protruding $\mathrm{Ce}^{4+}$ and two faint $\mathrm{Ce}^{3+}$ ions [Fig. 4(d)]. However, this configuration has not been detected in the experiment.

In conclusion, STM imaging and spectroscopy in combination with DFT calculations provide an unique insight into the localization of the two excess electrons that remain in the $\mathrm{CeO}_{2}$ (111) surface upon $\mathrm{O}$ vacancy formation. Although the precise position of the two $\mathrm{Ce}^{3+}$ ions is not revealed, we have demonstrated that they always sit in different coordination spheres around the defect, in agreement with earlier theoretical results [11]. The driving force for adopting such open configurations is the better ability of the system to relax lattice strain induced by the more spacious $\mathrm{Ce}^{3+}$ ion compared to its $\mathrm{Ce}^{4+}$ counterpart. Our results bring the long-standing puzzle on electron localization in defective $\mathrm{CeO}_{2}$ surfaces closer to a final answer.

We acknowledge financial support from the DFG within the Cluster of Excellence "UniCat" and the HLRN and thank J. Paier for various discussions.
*Corresponding author.

nilius@fhi-berlin.mpg.de

${ }^{\dagger}$ Present address: Institute of Catalysis and PetrochemistryCSIC, Marie Curie 2, 28049 Madrid, Spain.

Corresponding author. js@ chemie.hu-berlin.de

[1] A. Trovarelli, Catalysis by Ceria and Related Materials (World Scientific, Singapore, 2002).

[2] Q. Fu, H. Saltsburg, and M. Flytzani-Stephanopoulos, Science 301, 935 (2003).

[3] J. B. Park et al., J. Am. Chem. Soc. 132, 356 (2010).

[4] N. V. Skorodumova, S. I. Simak, B. I. Lundqvist, I. A. Abrikosov, and B. Johansson, Phys. Rev. Lett. 89, 166601 (2002).

[5] A. Pfau and K.D. Schierbaum, Surf. Sci. 321, 71 (1994).

[6] P. S. Bagus et al., Chem. Phys. Lett. 487, 237 (2010).

[7] F. Esch et al., Science 309, 752 (2005).

[8] S. Torbrügge, M. Reichling, A. Ishiyama, S. Morita, and Ó. Custance, Phys. Rev. Lett. 99, 056101 (2007).

[9] S. Fabris, G. Vicario, G. Balducci, S. de Gironcoli, and S. Baroni, J. Phys. Chem. B 109, 22860 (2005).

[10] M. Nolan, S. C. Parker, and G. W. Watson, Surf. Sci. 595, 223 (2005).

[11] M. V. Ganduglia-Pirovano, J. L. F. DaSilva, and J. Sauer, Phys. Rev. Lett. 102, 026101 (2009).

[12] H. Y. Li, H.F. Wang, X.-Q. Gong, Y.-L. Guo, Y. Guo, G. Lu, and P. Hu, Phys. Rev. B 79, 193401 (2009).

[13] C. Zhang, A. Michaelides, D. A. King, and S. J. Jenkins, Phys. Rev. B 79, 075433 (2009).

[14] C. Loschen, S. T. Bromley, K. M. Neyman, and F. Illas, J. Phys. Chem. C 111, 10142 (2007); A. Migani, K. M. Neyman, F. Illas, and S. Bromley, J. Chem. Phys. 131, 064701 (2009).

[15] M. Baron et al., Angew. Chem., Int. Ed. 48, 8006 (2009); F. Yang et al., J. Am. Chem. Soc. 133, 3444 (2011).

[16] J.-L. Lu, H.-J. Gao, S. Shaikhutdinov, and H.-J. Freund, Surf. Sci. 600, 5004 (2006); Catal. Lett. 114, 8 (2007).

[17] E. Wuilloud, B. Delley, W. D. Schneider, and Y. Baer, Phys. Rev. Lett. 53, 202 (1984).

[18] N. Nilius, T. M. Wallis, and W. Ho, Phys. Rev. Lett. 90, 046808 (2003).

[19] M. Grobis, A. Wachowiak, R. Yamachika, and M.F. Crommie, Appl. Phys. Lett. 86, 204102 (2005).

[20] We note that no spontaneous ceria reduction via the $\mathrm{Ru}$ support takes place as shown in Ref. [15].

[21] J. P. Perdew, J. A. Chevary, S. H. Vosko, K. A. Jackson, M. R. Pederson, D. J. Singh, and C. Fiolhais, Phys. Rev. B 46, 6671 (1992); G. Kresse and J. Furthmüller, Comput. Mater. Sci. 6, 15 (1996).

[22] A. M. Burow, M. Sierka, J. Döbler, and J. Sauer, J. Chem. Phys. 130, 174710 (2009). 